\title{
Radio Emission from the Intermediate-mass Black Hole in the Globular Cluster G1
}

\author{
James S. Ulvestad \\ National Radio Astronomy Observatory \\ P.O. Box O, Socorro, NM 87801 \\ julvesta@nrao.edu \\ Jenny E. Greene ${ }^{1}$ \\ Department of Astronomy, Princeton University \\ Princeton, NJ \\ jgreene@astro.princeton.edu \\ and \\ Luis C. Ho \\ The Observatories of the Carnegie Institute of Washington \\ 813 Santa Barbara St., Pasadena, CA 91101 \\ lho@ociw.edu
}

\begin{abstract}
We have used the Very Large Array (VLA) to search for radio emission from the globular cluster G1 (Mayall-II) in M31. G1 has been reported by Gebhardt et al. to contain an intermediate-mass black hole (IMBH) with a mass of $\sim 2 \times 10^{4} M_{\odot}$. Radio emission was detected within an arcsecond of the cluster center with an $8.4 \mathrm{GHz}$ power of $2 \times 10^{15} \mathrm{~W} \mathrm{~Hz}^{-1}$. The radio/X-ray ratio of G1 is a few hundred times higher than that expected for a high-mass X-ray binary in the cluster center, but is consistent with the expected value for accretion onto an $\mathrm{IMBH}$ with the reported mass. A pulsar wind nebula is also a possible candidate
\end{abstract}

\footnotetext{
${ }^{1}$ Hubble Fellow
} 
for the radio and X-ray emission from G1; future high-sensitivity VLBI observations might distinguish between this possibility and an IMBH. If the radio source is an IMBH, and similar accretion and outflow processes occur for hypothesized $\sim 1000 M_{\odot}$ black holes in Milky Way globular clusters, they are within reach of the current VLA and should be detectable easily by the Expanded VLA when it comes on line in 2010.

Subject headings: accretion, accretion disks — galaxies: individual (M31) — globular clusters: individual (G1) — radio continuum: galaxies

\section{Introduction}

Most galaxies with massive spheroidal components appear to harbor central black holes (BHs), with masses ranging from a few $\times 10^{6} M_{\odot}$ to over $10^{9} M_{\odot}$. These $\mathrm{BH}$ masses are well correlated with both the luminosity and the velocity dispersion of the galaxy spheroid (Gebhardt et al. 2000; Ferrarese \& Merritt 2000), implying that the formation of the central $\mathrm{BHs}$ is connected intimately with the development of the galaxy bulges. However, a bulge is not necessarily a prerequisite for a massive $\mathrm{BH}$. On the one hand, neither the late-type spiral galaxy M33 (Gebhardt et al. 2001) nor the dwarf spheroidal galaxy NGC 205 (Valluri et al. 2005) shows dynamical evidence for a massive BH. On the other hand, both the Sd spiral galaxy NGC 4395 and the dwarf spheroidal galaxy POX 52 contain active BHs with masses $\approx 10^{5} M_{\odot}$, although neither contains a classic bulge (Fillipenko \& Ho 2003; Barth et al. 2004). Greene \& Ho $(2004,2007)$ find that optically active intermediate-mass BHs (IMBHs, $M_{\mathrm{BH}}<10^{6} M_{\odot}$ ), while rare, do exist in dwarf galaxies, but optical searches are heavily biased toward sources accreting at high Eddington rates. Alternate search techniques are needed to probe the full demographics of IMBHs.

Direct dynamical detection of IMBHs is currently impossible outside of the Local Group. However, Gebhardt, Rich, \& Ho $(2002,2005)$ have found dynamical evidence for an excess dark mass of $(1.8 \pm 0.5) \times 10^{4} M_{\odot}$ at the center of the globular cluster G1 in M31; the evidence for this IMBH was questioned by Baumgardt et al. (2003), but supported by the improved data and analysis of Gebhardt et al. (2005). The physical nature of the central dark object is difficult to prove: it could be either an $\mathrm{IMBH}$ or a cluster of stellar remnants. While the putative presence of a BH in a globular cluster center may appear unrelated to galaxy bulges, the properties of G1, including its large mass, high degree of rotational support, and multi-aged stellar populations, all suggest that G1 is actually the nucleus of a stripped dwarf galaxy (Meylan et al. 2001). Most intriguingly, the inferred BH mass for G1 is about 0.1\% of the total mass, consistent with the relation seen for higher mass BHs, and consistent with 
predictions based on mergers of BHs (Miller \& Hamilton 2002) or stellar mergers in dense clusters (Portegies Zwart \& McMillan 2002).

Recently, Pooley \& Rappaport (2006) reported an X-ray detection of G1, with a $0.2-$ $10 \mathrm{keV}$ luminosity of $L_{\mathrm{X}} \approx 2 \times 10^{36} \mathrm{ergs} \mathrm{s}^{-1}$. Although this may represent accretion onto a central $\mathrm{BH}$, it is within the range expected for either accretion onto an IMBH or for a massive X-ray binary. Unfortunately, the most accurate X-ray position determined recently by Kong (2007) does not have sufficient accuracy to determine whether the X-ray source is located within the central core of G1, which would help distinguish between these two possibilities. The radio/X-ray ratio of G1 provides an additional test of the nature of the G1 X-ray source. As pointed out by Maccarone (2004) and Maccarone, Fender, \& Tzioumis (2005), deep radio searches may be a very effective way to detect IMBHs in globular clusters and related objects, since, for a given X-ray luminosity, stellar mass BHs produce far less radio luminosity than supermassive BHs. The relation between BH mass, and X-ray and radio luminosity empirically appears to follow a "fundamental plane," in which the ratio of radio to X-ray luminosity increases as the $\sim 0.8$ power of the $\mathrm{BH}$ mass (Merloni, Heinz, \& di Matteo 2003; Falcke, Körding, \& Markoff 2004). For an IMBH mass of $1.8 \times 10^{4} M_{\odot}$ in G1, one thus would expect a radio/X-ray ratio about 400 times higher than for a $10 M_{\odot}$ stellar BH. In this paper, we report a deep Very Large Array (VLA) integration on G1 and a radio detection that apparently confirms the presence of an IMBH whose mass is consistent with that found by Gebhardt et al. (2002, 2005).

\section{Observations and Imaging}

We obtained a 20-hr observation of G1 using the VLA in its C configuration (maximum baseline length of $3.5 \mathrm{~km}$ ) at $8.46 \mathrm{GHz}$. The observation was split into two 10-hr sessions, one each on 2006 November 24/25 and 2006 November 25/26. Each day's observation consisted of repeated cycles of 1.4 minutes observation on the local phase calibrator J0038+4137 and 6 minutes observation on the target source G1. In addition, each day contained two short observations of 3C $48(\mathrm{~J} 0137+3309)$ that were used to calibrate the flux density scale to that of Baars et al. (1977). Thus, the total integration time on G1 was $14.1 \mathrm{hr}$. We also obtained a total of $9.5 \mathrm{hr}$ of observing in C configuration at $4.86 \mathrm{GHz}$ on 2007 January 13/14 and 2007 January 14/15, using a similar observing strategy, and achieving a total of $7.3 \mathrm{hr}$ of integration on source.

All data calibration was carried out in NRAO's Astronomical Image Processing System (Greisen 2003). Absolute antenna gains were determined by the 3C 48 observations, then transferred to J0038+4137, which was found to have respective flux densities of $0.52 \mathrm{mJy}$ and 
$0.53 \mathrm{mJy}$ at 8.4 and $4.9 \mathrm{GHz}$. In turn, J0038+4137 was used to calibrate the interferometer amplitudes and phases for the target source, G1. Erroneous data were flagged by using consistency of the gain solutions as a guide and by discarding outlying amplitude points.

The VLA presently is being replaced gradually by the Expanded VLA (EVLA), which includes complete replacement of virtually all the electronic systems on the telescopes. Since antennas are refurbished one at a time, the VLA at the time of our observations consisted of 18-20 "old" VLA antennas and 6 "new" (actually, refurbished) EVLA antennas, having completely different electronics systems. Although all antennas were cross-correlated for our observations, we found subtle errors in some of the EVLA data. Thus, to be conservative, we discarded the data from all EVLA antennas except for 3 antennas that were confirmed to work very well on 2006 November 24/25.

The radio data were Fourier transformed and total-intensity images were produced in each band, covering areas of $17^{\prime} \times 17^{\prime}$ at each frequency 2 These images were CLEANed in order to produce the final images. At $8.4 \mathrm{GHz}$, the rms noise was $6.2 \mu \mathrm{Jy}_{\text {beam }}{ }^{-1}$ for a beam size of $2^{\prime \prime} .94 \times 2$ '. 72 ; at $4.9 \mathrm{GHz}$, the noise was $15.0 \mu \mathrm{Jy}_{\text {beam }}{ }^{-1}$ for a beam size of $5^{\prime \prime} .09 \times 4^{\prime \prime} .43$.

A few radio sources with strengths of hundreds of microjansky to a few millijansky were found in the images, but we discuss only G1 in this Letter 3 At $8.4 \mathrm{GHz}$, an apparent source with a flux density of $28 \pm 6 \mu \mathrm{Jy}$ [corresponding to $2 \times 10^{15} \mathrm{~W} \mathrm{~Hz}^{-1}$ for distance modulus of $(m-M)=24.42 \mathrm{mag}$ (Meylan et al. 2001)] was found approximately one arcsecond from the G1 optical position reported by Meylan et al. (2001); this radio source has J2000 coordinates of $\alpha=00^{h} 32^{m} 46.54^{s}, \delta=39^{\circ} 34^{\prime} 39.2^{\prime \prime}$. Figure 1 shows our $8.4 \mathrm{GHz}$ image of the $20^{\prime \prime}$ by $20^{\prime \prime}$ region centered on G1; this image includes a 1 sigma error circle of 1 ."5 radius for the X-ray position found by Kong (2007). The radio position has an estimated error of 0 '.6 in each dimension (not shown in the figure), derived by dividing the beam size by the signal-to-noise ratio 4

The a priori probability of finding a $4.5 \sigma$ noise spike or background source so close to G1 is quite small, as indicated by the lack of any other contours of similar strength in

\footnotetext{
${ }^{2}$ The image areas covered were much larger than the primary beams of the individual VLA antennas, in order to provide the best possible subtraction of confusing sources.

${ }^{3}$ The strongest nearby radio source is a $1 \mathrm{mJy}$ (at $8.4 \mathrm{GHz}$ ) object located approximately 100 " from G1; this object and the other weak sources might be X-ray binaries or supernova remnants if located at the distance of M31, but their numbers also are consistent with the possibility that some could be background extragalactic sources.

${ }^{4}$ Transfer of the phase from the local phase calibrator makes an insignificant contribution to the radio source position error, relative to the error imposed by the limited signal strength.
} 
Figure 1. If we hypothesize that there are 9 independent beams (roughly $8^{\prime \prime}$ by $8^{\prime \prime}$ ) within which a source would be considered to be associated with G1, then the probability of a $4.5 \sigma$ noise point close to G1 is less than $10^{-4}$. Similarly, the expected density of extragalactic radio sources at $28 \mu \mathrm{Jy}$ or above is $0.25 \mathrm{arcmin}^{-2}$ (Windhorst et al. 1993), or $4 \times 10^{-3}$ in a box $8^{\prime \prime}$ on a side, making it unlikely that we have found an unrelated background source. In order to search for possible data errors that might cause a spurious source, we have subjected our data set to additional tests, imaging data from the two days separately, and also imaging the two different intermediate frequency channels separately. The G1 radio source remains in the images made from each data subset, with approximately the same flux density and position. The overall significance is reduced by $2^{1 / 2}$ to approximately $3 \sigma$ in each image made with about half the data, as expected for a real source with uncontaminated data. Other $2.5 \sigma-3 \sigma$ sources appear in the central $20^{\prime \prime}$ box in some subsets of half the data, consistent with noise statistics, but none is above the $3.5 \sigma$ level in the full data set. Thus, all tests indicate that the detection of G1 is real, and we will proceed on that basis for the remainder of this paper. At $4.9 \mathrm{GHz}$, we find no detection at the G1 position, but the much higher noise level provides us only with very loose constraints on the source spectrum (see below).

\section{Origin of the G1 Radio Emission}

Merloni et al. (2003) and Falcke et al. (2004) have quantified an empirical relation (or "fundamental plane") among X-ray and $5 \mathrm{GHz}$ radio luminosity and BH mass; we use the Merloni et al. (2003) relation $L_{R} \propto L_{\mathrm{X}}^{0.6} M_{\mathrm{BH}}^{0.78}$. Merloni et al. (2003) analyzed this relation in the context of accretion flows and jets associated with massive BHs. One might expect some general relationship among these three quantities, if an X-ray-emitting accretion flow onto a massive BH leads to creation of a synchrotron-emitting radio jet, with the detailed correlation providing some insight into the nature of that flow. By comparing the empirically determined relation with expectations from theoretical models, Merloni et al. (2003) deduced that the data for BHs emitting at only a few percent of the Eddington rate are consistent with radiatively inefficient accretion flows and a synchrotron jet, but inconsistent with standard disk accretion models.

Maccarone (2004) scaled the fundamental-plane relation to values appropriate for an IMBH in a Galactic globular cluster; we rescale their equation here to find a predicted radio flux density of

$$
S_{5 \mathrm{GHz}}=52\left(\frac{L_{\mathrm{X}}}{10^{36} \mathrm{ergs} \mathrm{s}^{-1}}\right)^{0.6}\left(\frac{M_{\mathrm{BH}}}{10^{4} M_{\odot}}\right)^{0.78}\left(\frac{d}{600 \mathrm{kpc}}\right)^{-2} \mu \mathrm{Jy}
$$


Using the previously cited X-ray luminosity and BH mass for G1 and our adopted distance modulus, this predicts a $5 \mathrm{GHz}$ flux density of $77 \mu \mathrm{Jy}$ for G1. However, taking into account the $30 \%$ uncertainty in the IMBH mass, the unknown spectral index of the radio emission, and the dispersion of 0.88 in $\log L_{R}$ (Merloni et al. 2003), the predicted $8.4 \mathrm{GHz}$ flux density for G1 is in the range of tens to a few hundred microjansky. Thus, our radio detection of $28 \mu \mathrm{Jy}$ at $8.4 \mathrm{GHz}$ is consistent with the predictions for a $1.8 \times 10^{4} M_{\odot} \mathrm{IMBH}$, but strongly inconsistent with a $10 M_{\odot}$ BH. Since neutron star X-ray binaries in a variety of states have radio/X-ray ratios much lower than BH X-ray binaries (Migliari \& Fender 2006), and thus another 2 orders of magnitude below the observed value, stellar-mass X-ray binaries of any type are ruled out as the possible origin of the radio emission in G1.

We can use the radio/X-ray ratio to assess other possible origins for the radio emission. Here, we use the ratio $R_{\mathrm{X}}=\nu L_{\nu}(8.4 \mathrm{GHz}) / L_{\mathrm{X}}(2-10 \mathrm{keV})$ as a fiducial marker. For G1, $R_{\mathrm{X}} \approx 5 \times 10^{-5}$, which is considerably lower than $R_{\mathrm{X}} \approx 10^{-2}$ that is common to the Galactic supernova remnant Cas A, low-luminosity active galactic nuclei (supposing G1 might be a stripped dwarf elliptical galaxy), and most ultraluminous X-ray sources (cf. Table 2 of Neff, Ulvestad, \& Campion [2003], and references therein).

It is of interest to compare the G1 source to various relatives of pulsars as well. For instance, G1 is within the wide range of both luminosity and radio/X-ray ratio observed for pulsar wind nebulae (PWNs) (Frail \& Scharringhausen 1997), less luminous than the putative PWN in M81 (Bietenholz, Bartel, \& Rupen 2004), but considerably more luminous than standard pulsars or anomalous X-ray pulsars (Halpern et al. 2005). The $8.4 \mathrm{GHz}$ luminosity of G1 is similar to that of the magnetar SGR 1806 - 20 about 10 days after its outburst in late 2004, and the lack of a $4.9 \mathrm{GHz}$ detection would be consistent with the fading of SGR 1806 - 20 two months after the outburst (Gaensler et al. 2005). However, there is no published evidence for a gamma-ray outburst from G1, and the relatively steady apparent X-ray flux (Pooley \& Rappaport 2006) also argues against a transient source. Thus, the only stellar-mass object that might account for the radio and X-ray emission would be a PWN; using the scaling law given by Frail \& Scharringhausen (1997), we find a likely size of $\sim 10$ milliarcseconds for a PWN radio source in G1, implying that high-sensitivity VLBI observations could distinguish between a PWN and IMBH origin for the radio emission from G1.

Knowledge of the radio spectrum of G1 could provide more clues to the character of the radio emission, although either a PWN or an IMBH accretion flow might have a flat spectrum. In any case, our $5 \mathrm{GHz}$ observation simply is not deep enough. If we choose a $2 \sigma$ upper limit of $30.0 \mu \mathrm{Jy}$ at $4.9 \mathrm{GHz}(2 \sigma$ chosen since we know the position of the 8.4 GHz source with high accuracy), we derive a spectral index limit of $\alpha>-0.12 \pm 0.99$ 
(for $S_{\nu} \propto \nu^{+\alpha}, 1 \sigma$ error in spectral index), which has little power to discriminate among models.

The X-ray emission from G1 may be due to Bondi accretion on the IMBH, either from ambient cluster gas or from stellar winds (Pooley \& Rappaport 2006). Ho, Terashima, \& Okajima (2003) and Pooley \& Rappaport (2006) give approximate relations for the Bondi accretion on an IMBH in a globular cluster; for an ambient density of $0.1 \mathrm{~cm}^{-3}$, an ambient speed of $15 \mathrm{~km} \mathrm{~s}^{-1}$ for the gas particles relative to the $\mathrm{IMBH}$, and a radiative efficiency of $10 \%$, the Bondi accretion luminosity for the G1 IMBH would be $\sim 3 \times 10^{38} \mathrm{ergs} \mathrm{s}^{-1}$. The X-ray luminosity of $2 \times 10^{36} \mathrm{ergs} \mathrm{s}^{-1}$ measured by Pooley \& Rappaport (2006) thus implies accretion at just under $1 \%$ of the Bondi rate. Given that $L_{\mathrm{X}} / L_{\mathrm{Edd}} \approx 10^{-6}$, a more likely scenario is that G1 accretes at closer to $10 \%$ of the Bondi rate but with a radiative efficiency under $1 \%$. In this context, we note that the radio/X-ray ratio for G1 is $\log R_{\mathrm{X}}>-4.3$, which is above the value of -4.5 used to divide radio-quiet from radio-loud objects (Terashima \& Wilson 2003) 5 G1 therefore should be considered radio-loud, as inferred for BHs in galactic nuclei that radiate well below their Eddington luminosities (Ho 2002).

If the globular clusters in our own Galaxy also have central BHs that are $0.1 \%$ of their total masses, and they accrete and radiate in the same way as G1, many would have expected $5 \mathrm{GHz}$ radio flux densities in the 20-100 $\mu \mathrm{Jy}$ range; flux densities often would be in the 1$10 \mu \mathrm{Jy}$ range even for less efficient accretion and radiation (Maccarone et al. 2005). As Maccarone et al. (2005) summarize, there are few radio images of globular clusters that go deep enough to test this possibility. Fender (2004) points out that the Square Kilometer Array (SKA) will be able to test for the existence of IMBHs in many globular clusters. However, based on our results for G1, we suggest that it is not necessary to wait for the SKA; the current VLA can reach the hypothesized flux densities with some effort. The EVLA (Ulvestad et al. 2006), scheduled to be on line in about 2010, will have 40 times the bandwidth and 6.3 times the sensitivity of the current VLA in the frequency range near $8 \mathrm{GHz}$. This will enable the EVLA to reach the $1 \mu \mathrm{Jy}$ noise level in approximately 12 hours of integration, thus probing the range of radio emission predicted by Maccarone et al. (2005) for many globular clusters.

\footnotetext{
${ }^{5} \mathrm{~A}$ lower limit is given for $R_{\mathrm{X}}$ because this quantity traditionally is given in terms of the $2-10 \mathrm{keV}$ luminosity, whereas $\log R_{\mathrm{X}}=-4.3$ would correspond to the value computed for the $0.2-10 \mathrm{keV}$ luminosity given by Poolev \& Rappaport (2006).
} 


\section{Summary}

We have detected faint radio emission from the object G1, a globular cluster or stripped dwarf elliptical galaxy in M31. The emission has an $8.4 \mathrm{GHz}$ power of $2 \times 10^{15} \mathrm{~W} \mathrm{~Hz}^{-1}$. Assuming that the radio source is associated with the X-ray source in G1 (Pooley \& Rappaport 2006), the radio/X-ray ratio is consistent with the value expected for an accreting $\sim 2 \times$ $10^{4} M_{\odot}$ BH. Thus, the radio detection lends support to the presence of such an IMBH within G1. The other possible explanation, a pulsar wind nebula, could be tested by making very high-sensitivity VLBI observations of G1.

The National Radio Astronomy Observatory is a facility of the National Science Foundation operated under cooperative agreement by Associated Universities, Inc. We thank the staff of the VLA that made these observations possible. Support for JEG was provided by NASA through Hubble Fellowship grant HF-01196, and LCH acknowledges support from NASA grant HST-GO-09767.02. Both were awarded by the Space Telescope Science Institute, which is operated by the Association of Universities for Research in Astronomy, Inc., for NASA, under contract NAS 5-26555. We also thank Dale Frail for useful discussions about pulsar wind nebulae, and an anonymous referee for useful suggestions.

Facilities: VLA.

\section{REFERENCES}

Baars, J. W. M., Genzel, R., Pauliny-Toth, I. I. K., \& Witzel, A. 1977, A\&A, 61, 99

Barth, A. J., Ho, L. C., Rutledge, R. E., \& Sargent, W. L. W. 2004, ApJ, 607, 90

Baumgardt, H., Makiro, J., Hut, P., McMillan, S., \& Portegies Zwart, S. 2003, ApJ, 589, L25

Bietenholz, M. F., Bartel, N., \& Rupen, M. P. 2004, Science, 304, 1947

Falcke, H., Körding, E., \& Markoff, S. 2004, A\&A, 414, 895

Fender, R. 2004, New A Rev., 48, 1399

Ferrarese, L., \& Merritt, D. 2000, ApJ539, L9

Filippenko, A. V., \& Ho, L. C. 2003, ApJ, 588, L13

Frail, D. A., \& Scharringhausen, B. R. 1997, ApJ, 480, 364 
Gaensler, B. M., et al. 2005, Nature, 434, 1104

Gebhardt, K., et al. 2000, ApJ, 539, L13

Gebhardt, K., et al. 2001, AJ, 122, 2469

Gebhardt, K., Rich, R. M., \& Ho, L. C. 2002, ApJ, 578, L41

-.2005, ApJ, 634, 1093

Greene, J. E., \& Ho, L. C. 2004, ApJ, 610, 722

- 2007, ApJ, submitted

Greisen, E. W. 2003, in Information Handling in Astronomy - Historical Vistas, Astrophysics and Space Science Library Vol. 285, ed. A. Heck (Dordrecht: Kluwer), 109

Halpern, J. P., Gotthelf, E. V., Becker, R. H., Helfand, D. J., \& White, R. L. 2005, ApJ, 632, L29

Ho, L. C. 2002, ApJ, 564, 120

Ho, L. C., Terashima, Y., \& Okajima, T. 2003, ApJ, 587, L35

Kong, A. K. H. 2007, ApJ, in press, astro-ph/0703662

Maccarone, T. J. 2004, MNRAS, 351, 1049

Maccarone, T. J., Fender, R. P., \& Tzioumis, A. K. 2005, Ap\&SS, 300, 239

Merloni, A., Heinz, S., \& di Matteo, T. 2003, MNRAS, 345, 1057

Meylan, G., Sarajedini, A., Jablonka, P., Djorgovski, S. G., Bridges, T., \& Rich, R. M. 2001, AJ, 122,830

Migliari, S., \& Fender, R. P. 2006, MNRAS, 366, 79

Miller, M. C., \& Hamilton, D. P. 2002, MNRAS, 330, 232

Neff, S. G., Ulvestad, J. S., \& Campion, S. D. 2003, ApJ, 599, 1043

Pooley, D., \& Rappaport, S. 2006, ApJ, 644, L45

Portegies Zwart, S. F., \& McMillan, S. L. W. 2002, ApJ, 576, 899

Terashima, Y., \& Wilson, A. S. 2003. ApJ, 583, 145 
Ulvestad, J. S., Perley, R. A., McKinnon, M. M., Owen, F. N., Dewdney, P. E., \& Rodriguez, L. F. 2006, BAAS, 38, 135

Valluri, M., Ferrarese, L., Merritt, D., \& Joseph, C. L. 2005, ApJ, 628, 137

Windhorst, R. A., Fomalont, E. B., Partridge, R. B., \& Lowenthal, J. D. 1993, ApJ, 405, 498 


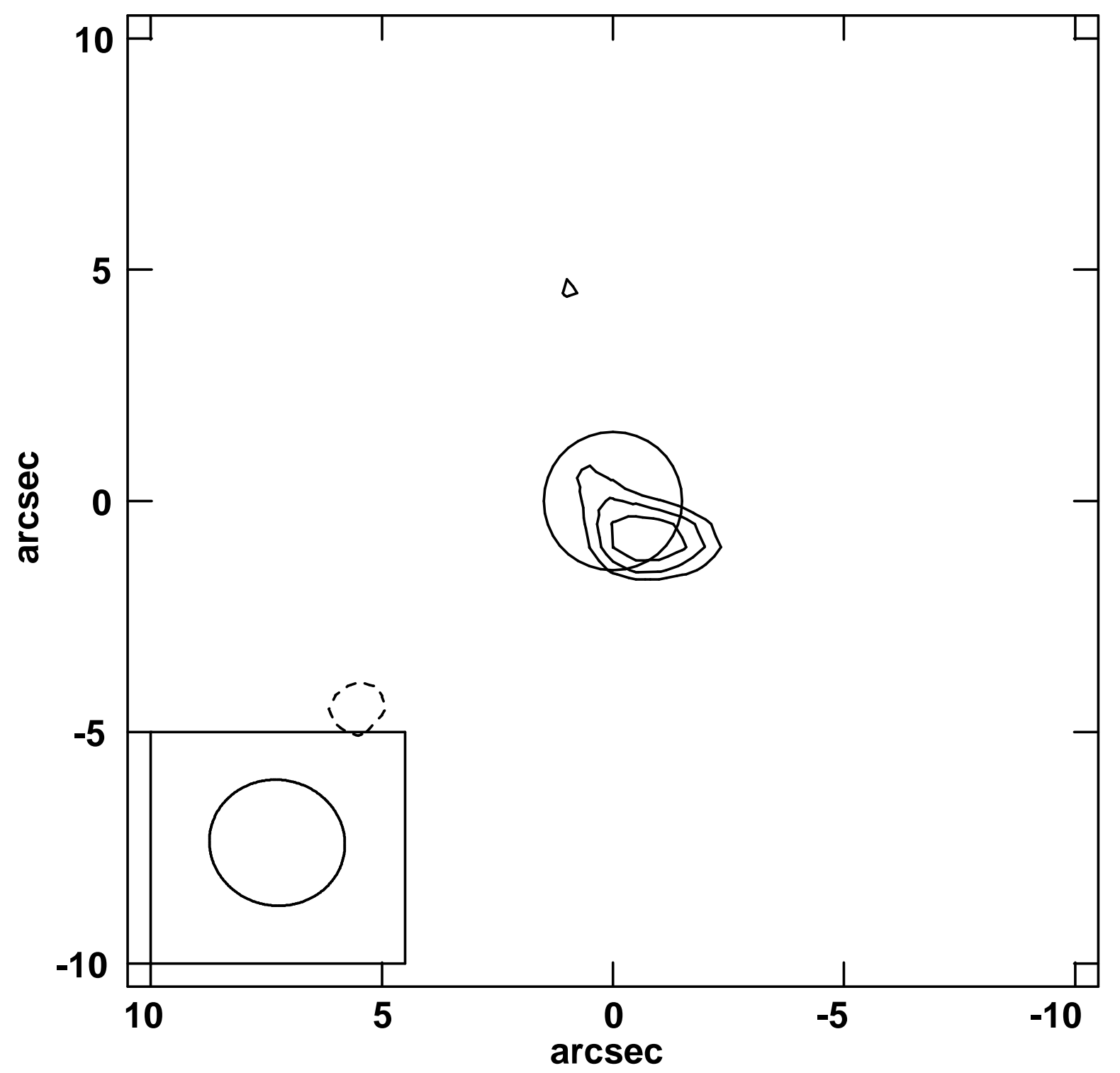

Fig. 1.- VLA C configuration $8.4 \mathrm{GHz}$ image of the vicinity of G1 in M31, with contours at intervals of 0.5 times the r.m.s noise. The lowest contour is at 3 times the noise of $6.2 \mu \mathrm{Jy}_{\text {beam }}{ }^{-1}$, and negative contours are shown dashed; the synthesized beam is shown in the box in the lower-left corner. The $(0,0)$ point is at a J2000 position of $\alpha=00^{h} 32^{m} 46.60^{s}$, $\delta=39^{\circ} 34^{\prime} 40.0^{\prime \prime}$. The $1^{\prime \prime} .5$-radius circle about this central point represents the $1 \sigma$ error in the X-ray position of G1 (Kong 2007). 